\title{
THE NATURE OF THE COMETARY KNOTS IN THE HELIX NEBULA AS DETERMINED BY HST IMAGES
}

\author{
C. R. O'DELL AND A. BURKERT \\ Max Planck Institute for Astronomy \\ Heidelberg, D-69117 GERMANY
}

\section{Introduction to and Global Properties of the Cometary Knots}

In a brief paper at the first meeting of this series, Vorontzov-Velyaminov (1968) presented the first examination of the fine scale structure found in the Helix Nebula. At that time he used the term "filaments" to describe them, although the results of improved spatial resolution during the ensuing decades (Malin 1982, Meaburn et al. 1992, Walsh \& Meaburn 1993) show that the term "Cometary Knot"(CK) communicates a clearer picture of their form. The most useful groundbased images are those of Meaburn et al.(1992) made with the New Technology Telescope, which showed that each CK has a characteristic form, composed of a central dark knot, accompanied by a luminous cusp on the substellar side, and accompanied by a thin sheath of luminous material extending from the edge of the cusp to large distances. Owing to their much higher density, the Cusps are of lower ionization than the nebula as a whole, so that one sees the central dark knots, which evidently contain significant amounts of dust, in silhouette against the background nebular emission. Radio observations of the CO line show that the CK are also sources of molecular emission (Huggins et al.1992). The groundbased emission line images show that the "tails" trailing away from the Cusps lie closely on radial lines passing between the Cusp and the Central Star. Although these objects appear to be diving towards the Central Star, such an interpretation is naive, as their form must be determined by a central repulsive force arising from the star, much as true comets in our Solar System may trail their tails behind while approaching the Sun but actually follow their tails as they recede from the Sun. We also now know, from yet another excellent study by Meaburn et al. (1996) that the CK as a group are expanding away from the Central Star, although this group velocity of about $10 \mathrm{~km} \mathrm{~s}^{-1}$ is about half that value of $21 \mathrm{~km} \mathrm{~s}^{-1}$ characteristic of the nebula as a whole (Taylor 


\section{COMETARY KNOTS IN THE HELIX NEBULA}

1977,Terrett 1979). The greatest breakthrough in imaging was the Hubble Space Telescope's WFPC2 (O'Dell \& Handron 1996, hereafter O\&H) program on a single field of view (FOV) in the north ring of the Helix in $\mathrm{H} \alpha$, [NII], and [OIII]. One year later the same field was reimaged in [OI] and with the $5547 \mathrm{~m}$ filter, the newer observations allowing calibration of the emission line images with correction for the underlying continuum. A second, contiguous field, was also imaged, in all five filters. The results of both sets of observations are used in this paper and a combined color image is shown in the rear section of this volume. The method of calibration was the same as that used in studies of the Orion Nebula (Hu 1993). Details of the calibration and digital copies of the images can be obtained from the first author. The new observations show heretofore unseen structure which is discussed in the following sections. They indicate that there are about 3500 detectable CK in the entire Helix Nebula, with the actual number probably being much larger as strong observational selection effects operate against detection of objects far from the Central Star. The images also show that the previously known orientation along radial lines is followed, but a detailed examination shows that the tails show small local variations, as if additional, non-radial, forces are acting on them (Fig.4). O\&H demonstrated that the chord diameters, measured across the cusps, decrease from about 1.8" at 120" distance from the Central Star to about 0.6 " at 180 ". There are so many known CK that one cannot use a sequential system of designation. In this paper we will use a coordinate based system, dividing the nebula into boxes of 1 " in Declination and $0.1^{s}$ of Right Ascension and dropping the values common to all of the CK. Therefore, a CK located at 22:29:42.331 -20:47:32.1 would have the designation Helix 423-732. This system will allow future studies to uniquely identify all CK with only six digits within the range of declination -20:45:00 and -20:55:00, with any objects found north or south of this range having either 4 or 5 inserted as an additional digit immediately after the hyphen. Epoch 2000 is used for the positions and the position is judged to be at the center of the dark knot.

The central star has been well studied, as befits the arguably closest of the Planetary Nebulae (PN). The paper of Hugh Harris in this conference reports that its parallax indicates a distance of $213 \mathrm{pc}$, a figure used in the remainder of this paper, but which is larger than the value of 150 adopted by $\mathrm{O} \& \mathrm{H}$. This change propagates through the analysis, so that many of the numbers cited below reflect the use of the improved distance. At this distance the WFPC2 pixel size of 0.0996 " projects to $3.17 \times 10^{14}$ $\mathrm{cm}$. The central star has been determined to have an effective temperature of $123,000 \mathrm{~K}$ (Bohlin et al. 1982) and an absolute luminosity of $120 L_{\text {sun }}$. No stellar wind features were detected in the study of Cerruti-Sola \& Perinotto 


\section{O'Dell}

(1985), which may indicate that the wind has dropped; however, the high
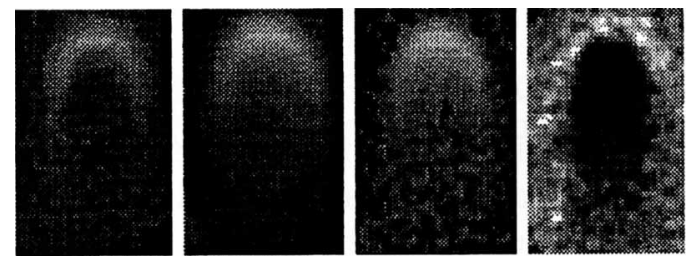

Figure 1. Images of the six averaged $\mathrm{CK}$ are shown in $\mathrm{H} \alpha$, [NII], [OI], and [OIII] (left to right). Each field is $3.3 \times 5.1 "$.

surface gravity of this white-dwarf-like central star indicates that any wind would be of very high velocity. This last fact could mean that a wind that is dynamically important could be present, but still falls beneath the threshold for spectroscopic detection (an important point to which we will return).

The Helix (NGC 7293, PK 36-57 ) is among the largest and oldest PN, its approximate angular radius of 6 ' corresponding to $0.37 \mathrm{pc}$ and a formal expansion age (size/expansion velocity) of 17,000 years. The density of the main ring derived from the $\mathrm{H} \alpha$ surface brightness and our derived value of the aspect ratio of 0.15 is only $60 \mathrm{~cm}^{-3}(\mathrm{O} \& \mathrm{H})$.

\section{Characteristics of Individual Cometary Knots}

There is a remarkable uniformity of appearance of the CK. A characteristic appearance is shown in Fig.1, where we have averaged six CK (351-802,352$815,354-804,378-800,428-900,474-931)$ all having apparent chord sizes of close to 1.7". Their inner appearances are very similar and the resulting average image of the six is of much higher signal to noise ratio than any individual. The greatest variation is in the central optical depth, which is probably due to the fact that some of the CK are located on the near side of the nebula. These CK give higher optical depths when compared with the local nebular emission, while others are on the far side and have much foreground emission present. All of the monochromatic images in Fig. 1 are simple averages of the surface brightnesses, except for the [OIII] image, where the objects were normalized to the local nebular surface brightness before averaging.

\section{Properties of the Cusps}

O\&H have shown that the Cusps are photoionized surfaces, optically thick to Lyman continuum radiation, on the face of a neutral CK, the peak surface 


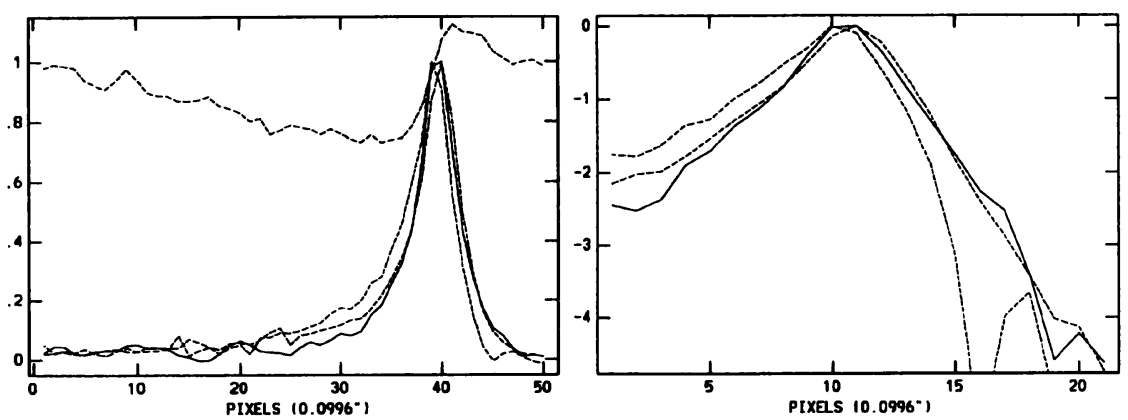

Figure 2. Two intensity plots of a central trace through the averaged CK. The left is linear intensity with the top line being the [OIII] emission normalized to the local nebular intensity. The three lower lines are intensity plots of [OI] (leftmost), $\mathrm{H} \alpha$ (broadest), and [NII]. The right hand shows a natural log plot of the intensities, normalized to the peak, and illustrating the rapid decay of [OI] emission and the nearly exponential decay of $\mathrm{H} \alpha$ and $[\mathrm{NII}]$ intensity.

brightness in $\mathrm{H} \alpha$ showing the expected decay with increasing Central Star distance. We have used the averaged images of Fig. 1 to determine detailed properties of the Cusps by taking a sample three pixels wide, centered on the $\mathrm{CK}$ and directed along a radius to the star. This sample represents a cut through the ionization front and one would expect to see differences in the appearance in different emission lines, the picture being complicated by the fact that we are actually sampling along lines of sight across a curved ionization front. However, since the peak emission will come from the region near the substellar point, which is tangential to our line of sight, a comparison with the expectations of simple ionization theory is possible. These samples are shown in Fig.2a and 2b. Since $\mathrm{H} \alpha$ emission is expected to arise from where-ever there is $\mathrm{HII}$, we can understand why the $\mathrm{H} \alpha$ emission peak is broadest and overlaps the others. [OI] emission requires both the presence of neutral Oxygen and energetic electrons normally produced by the ionization of hydrogen, conditions satisfied only in the ionization front proper. The fact that the [OI] emission falls on the left boundary of the emission is understood in this context. The width of this emission region can also be understood. We know from the $\mathrm{H} \alpha$ surface brightness that the peak density $\left(N_{e}\right)$ of the Cusps is $1200 \mathrm{~cm}^{-3}$. Using the absorption coefficient $(\kappa)$ at the peak of emission for the Central Star (Osterbrock 1989) gives an expected ionization front width of $(\kappa N)^{-1}$ of $9.2 \times 10^{14} \mathrm{~cm}$, which corresponds to 2.9 pixels. The observed with is about 5 pixels, which is good agreement since we expect the observed value to be increased by emission coming from the non-tangential photoionized region. The [NII] emission close to the ionization front. The [OIII] zone will lie farthest from the 


\section{O'Dell}

ionization front, which again agrees with the observations. In summary, we can say that the gross ionization properties agree well with the predictions of ionization of small knots.

$\mathrm{O} \& \mathrm{H}$ also argued that the brightness distribution dropped exponentially, a characteristic confirmed in Fig.2b, where an exponential decay will appear as a straight line. The observed scale height for the $\mathrm{H} \alpha$ surface brightness is 1.9 pixels, which corresponds to a density scale height of 3.8 pixels, or $1.2 \times 10^{15} \mathrm{~cm}$. If the emission is indeed dominated by radiation from the substellar point, then this argues that the HII region is an atmosphere in equilibrium with a constant force, rather than freely expanding. The source of this force is not obvious. Radiation pressure is insufficient by some four orders of magnitude to produce equilibrium. A stellar wind is a way out, but it would not act directly since its kinetic energy would be deposited at an inner shock which is theoretically expected to produce a low density inner region of about $10^{6} \mathrm{~K}$ (Kwok 1982, Kahn 1983, Volk $\& \mathrm{Kwan} 1985$ ). If the cusp electron temperature is $10^{4} \mathrm{~K}$, then an inner hot region density of only $N_{e}=12$ (at $T_{e}=10^{6} \mathrm{~K}$ ), would be required for equilibrium, which would be undetectably faint in optical emission.

Certainly one needs a radial repulsive force to explain the streaming out of material in the tails beyond the tips of the Cusps. One cannot explain the situation as due to an unknown force that has recently stopped, because the timescale for destruction of the tight compression by free flow is well less than 100 years. X-ray emission from a high temperature inner gas such as that we require to explain the size of the cusp atmospheres has been detected by ROSAT (Leahy et al. 1994); however, it was spatially unresolved and concentrated to the Central Star. Given the uncertainty of the mass loss rate and the temperature of the inner region, high temperature shocked gas appears to be the most promising explanation of the confinement of the Cusp atmospheres.

\section{Properties of the Neutral Cores}

The most important aspect of the CK is probably their neutral cores, even though they are much more difficult to study than their photoionized surfaces. As indicated above, the neutral cores manifest themselves by being seen in extinction against the background emission of the nebula and through their $\mathrm{CO}$ emission. They are well resolved and Fig. 3 shows the profile of an average of five objects, all of which are large and of nearly the same central extinction. We also show the results of calculating the column density of particles causing the extinction (using the previously adopted distance of $150 \mathrm{pc}$, these numbers needing to be multiplied by 2.0 according to the new distance of Harris announced at this symposium). There 
is no evidence of an extremely high density neutral core, but such a core

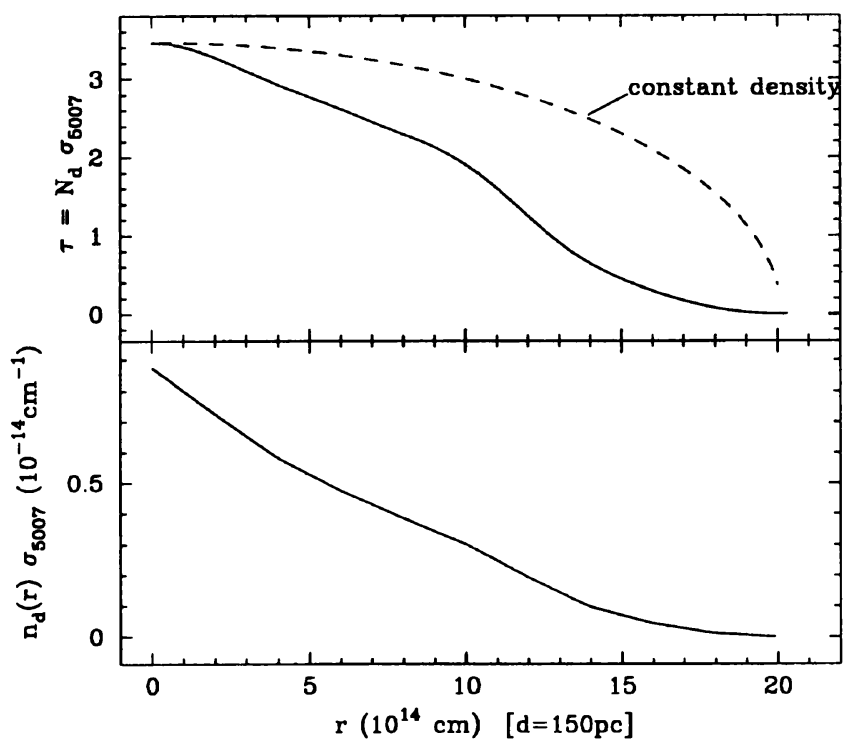

Figure 3. The upper part of this figure shows the variation of optical depth along the chord of a composite of five similar CK and the lower the derived column density of dust.

would be difficult to detect in measurements along lines-of-sight. One does see in Fig. 3 that there is a strong central concentration. If the gas to dust ratio is similar to the value of 150 in the general interstellar medium (ISM) (Sodroski et al. 1994), then the average density of these CK is $1.1 \times 10^{6} \mathrm{~cm}^{-3}$ and their characteristic mass about $3 \times 10^{-5} M_{\text {sun }}$. Since there appear to be about $3500 \mathrm{CK}$ in the Helix, then the total mass is about $0.1 \mathrm{M}_{\text {sun }}$, which is a significant fraction of the ionized mass of the entire nebula. We have used Fig. 6 of O\&H to determine the geometry of the Helix, with the result that the object is best approximated as a nearly flat on disk with a thickness equivalent to 220 ". This model was used for deriving the gas density, and also to determine a total mass of $0.11 M_{\text {sun }}$ for the main ring of the nebula. This near unity ratio of $\mathrm{CK}$ and ring material means that we are dealing with an important component of this nebula. Since this is the closest and best resolved PN, it raises the possibility that condensed knots are ubiquitous and therefore important in understanding the physics of the PN in general, a point previously made (Capriotti et al. 1971, Kirkpatrick 1972) outside of discussions of the Helix. Higher resolution observations with the Planetary Camera portion of WFPC2 would certainly help to clarify the characteristics of the $\mathrm{CK}$, but the most promising observations 


\section{O'Dell}

would be higher resolution studies in molecular lines and in the infrared thermal continuum.

\section{Origin and Future of the CK}

The origin and future of the CK are vital questions to resolve. Their origin may tell us about the physical processes in PN or even broader mechanisms, while their future can have impact on questions of the general composition of the ISM.

Capriotti (1973) originally argued that the CK result from RayleighTaylor instabilities arising at the ionization front of the nebula, which is an over-pressure situation with the hot inner ionized gas pushing against the cold neutral outer gas. It can be argued that the over-pressure conditions produced by a stellar wind can also produce instabilities, but Capriotti (1996) has repeated his simplified analytic solutions and found that instabilities of the original type would be more important.

We have also made a hydrodynamical simulation of Rayleigh-Taylor instabilities arising at the ionization boundary of a PN. The calculations were performed in two dimensions, using a second-order Eulerian grid code (Burkert \& Bodenheimer 1993) with 200x200 grid cells. In this model, the ionized, low-density inner region with temperature $T=10^{4} \mathrm{~K}$ pushes against the ionization boundary of a constant and high-density ring of neutral gas which initially is at rest. As a result, the neutral gas is swept up into a thin, partly ionized shell. Small density perturbations in the shell amplify exponentially due to Rayleigh-Taylor instabilities as long as the shell is accelerated outwards and produce filaments pointing towards the Central Star. The results are shown in Fig. 4 and can be compared with the observed CK. Although these fingers resemble the structures we see in the Helix Nebula their substructure differs from the observations in important ways. For example, the tips are completely ionized and the gas density increases systematically towards the main ionization front. The observations, on the other hand, show dense cometary knots at the tips of the filaments with the density decreasing outwards. Our calculations indicate that although Rayleigh-Taylor instability leads to filamentary structures within PN, the observed CK must have a different origin. More detailed high-resolution calculations which will take into account the complete mass-loss history of the Central Star, including a fast wind phase, as well as the time variation of the Central Star's UV photon flux will be reported elsewhere. It appears that the most likely origin is local density fluctuations which are optically thick to the Lyman continuum and remain behind as the main ionization front advances into the larger scale neutral material. This material would then be compressed by the surrounding high pressure, ionized gas, eventu- 


\section{COMETARY KNOTS IN THE HELIX NEBULA}

ally reaching pressure equilibrium, which O\&H and Meaburn et al.'s(1992) observations indicate is indeed the case. The possibility of the CK being
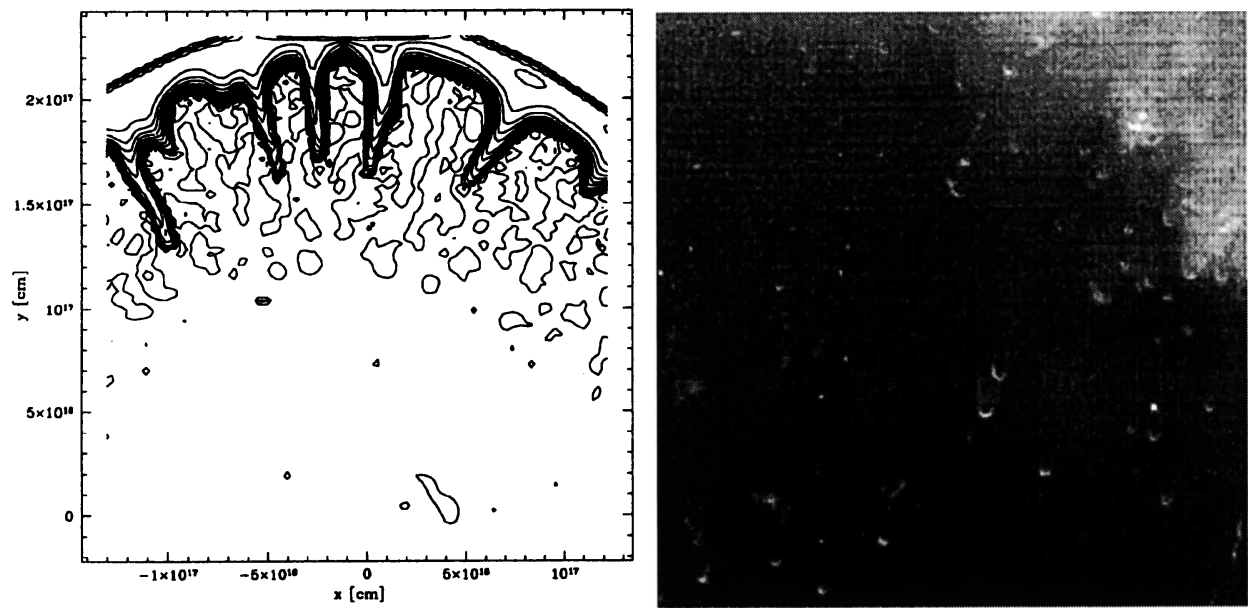

Figure 4. The left side shows iso-density contours of a model for Rayleigh-Taylor instabilities arising from the ionization boundary of a PN. The right side shows a composite of $\mathrm{H} \alpha$ and [NII] images out of the combined field,indicating the near commonality of orientation and symmetry.

primordial knots remaining from the formation of the Central Star or the manifestations of an Oort Cloud around that star are discussed in depth in O\&H and will not be repeated here. The appearance of the O\&H paper has brought out comparison with observational evidence for similar mass bodies in other galaxies (Schild 1996) and several cosmological models that would argue for the formation of such bodies early in the evolution of the universe (Brosche 1989, 1995; Gibson 1996).

The future of the CK is quite important. O\&H point out that there is sufficient material in the neutral portions to survive the effects of photoablation while the Central Star is still an important source of photoionizing radiation. Their density and size would appear to be insufficient to produce gravitational collapse and the question of their future requires the construction of theoretical models for the conditions that would prevail after the central star has cooled and become an unimportant source of energy. If the CK do eventually dissipate, their material will simply become part of the ISM, being important only as a region where a substantial part of the stellar mass that is being returned to the ISM was temporarily stored in a space where dust and molecules could have formed and survived. If the CK do survive, possibly as solid bodies, then the fact that they are known to have expansion velocities that far exceed the gravitational attraction 


\section{O'Dell}

of the Central Stars means that the ISM would have an important new component.

We wish to acknowledge with gratitude the aid of Kerry Patrick Handron in preparing the color image and figures 3 and 4 . The WFPC2 observations were made with the NASA/ESA Hubble Space Telescope, obtained at the Space Telescope Science Institute, which is operated by the Association of Universities for Research in Astronomy, Inc. under NASA Contract No. NAS 5-26555. Author O'Dell's research was supported by NASA grant NAG 5-1626 to the William Marsh Rice University.

\section{References}

Bohlin R.C., Harrington J.P., \& Stecher T.P. 1982,ApJ,252,635

Brosche P. 1989, A\&A, 219, 13

Brosche P. 1995, Astr.Nach, 316, 149

Burkert A. \& Bodenheimer P. 1993, MNRAS, 264, 798

Capriotti E.R. 1973, ApJ, 179, 495

Capriotti E.R. 1996, BAAS,in press

Capriotti E.R., Cromwell R.H., \& Wiliams R.E. 1971, ApJL, 7, 241

Cerruti-Sola M. \& Perinotto M. 1985, ApJ, 291, 237

Gibson C.H. 1996, App.Mech.Rvw., 49, no.5, 299

Hu Xihai 1993, STScI Newsletter, 10, no.2, 28

Huggins P.J., Bachiller R., Cox P., \& Forveille T., 1992, ApJ, 401, L43

Kahn F.D. 1983,in IAU Symp.103, Planetary Nebulae, ed.D.R.Flower (Dordrect: Reidel), 305

Kirkpatrick,R.C. 1972,ApJ,176,381

Kwok S. 1982, ApJ, 258, 280

Kwok S. \& Leahy D.A. 1984, ApJ, 283, 765

Leahy D.A., Zhang C.Y., \& Kwok S. 1993, ApJ, 422, 205

Meaburn J., Walsh J.R., Clegg R.E.S., Walton N.A., Taylor D., \& Berry,D.S. 1992, MNRAS, 255, 177

Meaburn J., Clayton C.A., Bryce M., \& Walsh J.R. 1996, MNRAS, 281, L57

Malin D.F. 1982, Sky\&Tel, January, 22

O'Dell C.R. \& Handron K.D. 1996, AJ, 111, 1630

Osterbrock D.E. 1989, Astrophysics of Gaseous Nebulae and Active Galactic Nuclei(Mill Valley,California: University Science Books)

Schild R.E., 1996, ApJ, 464, 125

Sodroski T.J., Bennett C., Boggess N., Dwek E., Franz B.A., Hauser M.G., Kelsall T., Moseley S.H., Odegard N., Silverberg R. F., Weiland J.L., 1994, ApJ, 438, 638

Taylor K., 1977, MNRAS, 181, 475

Terrett D.L., 1979, MNRAS, 186, 127

Vorontzov-Velyaminov B.A. 1968, in Planetary Nebulae:IAU Symposium 34, eds. D.E. Osterbrock \& C.R.O'Dell (Dordrecht:Reidel), 256

Walsh J.R. \& Meaburn J., 1993, Messenger, 73, 35 